\title{
An Improved PSO Algorithm Based on SA and Quantum Theory and Its Application
}

\author{
Wei Tan ${ }^{1}$, Shoubin Dong ${ }^{2}$, Xuan $\mathrm{Liu}^{3}$ and Bin Wang ${ }^{1}$ \\ ${ }^{1}$ Department of computer, Dongguan University of Technology, Dongguan, \\ 523808 Guangdong, China \\ ${ }^{2}$ School of Computer Science and Engineering, South China University of \\ Technology, Guangzhou 510006, Guangdong, China \\ ${ }^{3}$ Department of economic and trade, Dongguan University of Technology, \\ Dongguan, 523808 Guangdong, China
}

\begin{abstract}
Due to the low computational precision, local optimal solution and slow convergence speed of particle swarm optimization (PSO) algorithm, an improved PSO (SAQPSO) algorithm based on simulated annealing (SA) and quantum theory is proposed in this paper. The first, quantum theory is used to change the updating mode of the particles in order to improve the search speed and the convergence precision, and guarantee the simplification and effectiveness. Then the SA with probability and local search ability is introduced into quantum PSO (QPSO) in order to keep the diversity of the population, avoid falling into local optimum and enhance the global search ability. The SAQPSO algorithm keeps the characteristics of the simple and easy implementation, improves the global optimization ability and the convergence speed and the accuracy. Finally, some benchmark functions are used to prove the validity of the proposed SAQPSO algorithm. The computational results show that the proposed SAQPSO algorithm takes on the fast convergence speed, the better robustness and global search ability.
\end{abstract}

Keywords: Particle swarm optimization; simulated annealing; quantum theory; optimization; complex problem

\section{Introduction}

Particle swarm optimization (PSO) algorithm is a stochastic optimization algorithm, and was proposed by Kennedy and Eberhar in 1995 [1]. The PSO algorithm derives from the simulation of the bird population and fish foraging behavior. It has characteristics of easy describing, convenient realizing, less adjustment parameters, relatively smaller swarm, less evaluation function times for the convergence, fast convergence speed, parallel processing and good robustness and so on. And it can find the global optimal solution of solving problem by larger probability, and the calculation efficiency is higher than traditional random methods. So the PSO algorithm was proposed to immediately cause widespread concern in evolutionary computation field, and many searchers proposed a large number of research results in a few years. It has been successfully applied in the function optimization, neural network training, fuzzy system control, data classification, pattern recognition, signal processing, robot technology and so on[2].

The PSO algorithm has the superiority for solving high dimensional complex problems. However, when it encountered search space of local minimal points, it also will show its deficiencies, especially when particles are near to local optimal solution in the space, the search efficiency of population may suddenly reduce [3]. If a new mechanism is provided for the PSO algorithm in falling into local optimum, it can jump out local optimal location with a high probability, and search other area of the solution space. So simulated 
annealing (SA) and quantum theory are introduced into the PSO algorithm to change the updating mode of the particles for improving the search speed and the convergence precision, and keep the diversity of the population, avoid falling into local optimum and enhance the global search ability. An improved PSO (SAQPSO) algorithm based on simulated annealing (SA) and quantum theory is proposed in this paper.

\section{Related Works}

In allusion to the low computational precision, local optimal solution and slow convergence speed of the PSO algorithm, a lot of searchers proposed many improved PSO algorithms in order improve the optimization performance of the PSO algorithm. Xu et al. proposed an improved particle swarm optimization (IPSO) algorithm with the mutation in weighted gradient direction based on the evaluation of the fitness variance, which can avoid the shorts of easily getting in local extremum and easy in premature convergence [4]. Jiang et al. proposed an improved PSO algorithm for the optimization of short-term generation scheduling [5]. Luo et al. an improved particle swarm optimization (PSO) algorithm for solving non-convex NLP/MINLP problem with equality and/or inequality constraints [6]. Chuang et al. proposed an improved binary particle swarm optimization (IBPSO) to implement feature selection, and the K-nearest neighbor (K-NN) method serves as an evaluator of the IBPSO for gene expression data classification problems [7]. Yuan et al. an improved particle swarm optimization (IPSO) to solve DLED with valve-point effects [8]. Hota et al. presented an improved particle swarm optimization (IPSO) technique for the solution of optimal power generation to short-term hydrothermal scheduling problem [9]. The IPSO technique is suggested that deals with an inequality constraint treatment mechanism called as dynamic search-space squeezing strategy to accelerate the optimization process and simultaneously. Omkar et al. proposed a novel multi-objective optimization algorithm developed on the basis of the Quantum behaved Particle Swarm Optimization (QPSO) paradigm [10]. Niknam et al. proposed a hybrid evolutionary programming based clustering algorithm, called PSO-SA, by combining particle swarm optimization (PSO) and simulated annealing (SA) [11]. Behnamian and Fatemi Ghomi proposed a hybrid approach based on particle swarm optimization (PSO) and simulated annealing (SA) [12]. The hybridization of a PSO with SA, combining the advantages of these two individual components, is the key innovative aspect of the approach. Dong et al. proposed the chaos-PSO (COSPSO) algorithm by introducing the chaos optimization mechanism and a global particle stagnationdisturbance strategy into the basic PSO [13]. Abdel-Kader proposed a new adjustable PSO-GA hybrid multicast routing algorithm which combines PSO with genetic operators [14]. The proposed hybrid technique combines the strengths of PSO and GA to realize the balance between natural selection and good knowledge sharing to provide robust and efficient search of the solution space. Wang et al. presented an improved self-adaptive particle swarm optimization algorithm (ISAPSO) to solve hydrothermal scheduling (HS) problem [15]. Tajbakhsh et al. proposed a very fast hybrid metaheuristic algorithm based on combining particle swarm optimization (PSO) and simulated annealing (SA) [16]. Thanushkodi and Deeba proposed an improved PSO based on the AIS to achieve better solutions [17]. Gao et al. proposed improved particle swarm algorithm (PSO) to ensure the subdivision accuracy of the photoelectric rotary encoder and subdivision multiple of the Moire fringe photoelectric signal [18]. Behrooz et al. presented an improved territorial particle swarm optimization (TPSO) algorithm [19]. Li et al. proposed an improved particle swarm optimization (PSO) algorithm with a neighborhood-redispatch (NR) technique to design an ultrawideband (UWB) antenna [20]. Walid et al. proposed a novel approach by introducing a PSO, which is modified by the ACO algorithm to improve the performance [21]. Ji et al. proposed an improved PSO based on update strategy of double 
extreme value by analyzing the updating ways of double extreme [22]. Chen et al. proposed chaotic improved PSO-based multi-objective optimization (MOCIPSO) and improved PSO-based multi-objective optimization (MOIPSO) approaches for solving complex multi-objective, mixed integer nonlinear problems such as minimization of power losses and L index in power systems simultaneously [23]. Wu et al. proposed a dual-group interaction quantum-behaved particle swarm optimization (QPSO) algorithm based on random evaluation (DIR-QPSO) by constructing the master-slave sub-groups with different potential well centers, which avoids the rapid disappearance of swarm diversity and enhances the global searching ability through collaboration between subgroups [24].

\section{Quantum Theory, SA and PSO Algorithm}

\subsection{Simulated Annealing (SA)}

Simulated annealing (SA) algorithm was developed by Kirkpatrick [25], followed by Aarts and Korst based on the Metropolis algorithm from 1953. It is a computational stochastic technique in order to get near global optimum solutions. The SA is inspired from the thermodynamic process of annealing of molten metals to attain the lowest free energy state. When molten metal is slowly cooled, it tends to solidify in a structure of minimum energy. The key of SA algorithm is to allow the occasional worsening moving, so that these can eventually help locate the neighborhood to the global minimum. Boltzmann gave the associated expression of the probability.

$$
\operatorname{probability}(p)=\exp \left(\frac{-\Delta E}{K_{b} T}\right)
$$

In the expression (1), $K_{b}$ is a constant, $\Delta E$ is the change in the energy value from one point to the next, $T$ is the temperature.

For the energy term, $\Delta E$ refers to the function value, $\mathrm{T}$ is a control parameter that regulates the process of annealing. The acceptance criterion is popularly referred to as the Metropolis criterion. Galuber gave the improved acceptance criterion:

$$
\operatorname{probability}(p)=\frac{\exp (-\Delta E / T)}{1+\exp (-\Delta E / T)}
$$

\subsection{Particle Swarm Optimization (PSO) Algorithm}

The PSO algorithm is a search algorithm based on the simulation of the social behavior of birds within a flock [2]. The positions of particles within the search space are changed based on the social-psychological tendency of individuals. The changing of particle is influenced by the experience, or knowledge. The consequence of modeling is that the search is processed in order to return toward previously successful regions. Namely, the velocity $\left(V_{i}=\left(v_{i 1}, v_{i 2}, \cdots, v_{i n}\right)\right)$ and position $\left(X_{i}=\left(x_{i 1}, x_{i 2}, \cdots, x_{i n}\right)\right)$ of the $i^{\text {th }}$ particle will be changed by the particle best value (pbest) and global best value $($ gbest $)$. The velocity and position updating of the particle is shown by the followed expression:

$$
\begin{aligned}
& v_{i j}(t+1)=w v_{i j}(t)+c_{1} r_{1}\left(\text { pbest }_{i j}(t)-x_{i j}(t)\right)+c_{2} r_{2}\left(\text { gest }_{i j}(t)-x_{i j}(t)\right) \\
& x_{i j}(t+1)=x_{i j}(t)+v_{i j}(t+1)
\end{aligned}
$$


Where $v_{i j}(t+1)$, the velocity of particle $i^{\text {th }}$ at iterations $j^{t h}, x_{i j}(t+1)$, the position of particle $i^{\text {th }}$ at iterations $j^{\text {th }}$. $w$ is inertia weight $\mathrm{t}$ denotes the iteration number, $c_{1}$ is the cognition learning factor, $c_{2}$ is the social learning factor, $r_{1}$ and $r_{2}$ are random numbers uniformly distributed in [0-1].

\section{Quantum PSO (QPSO) Algorithm}

In the classical PSO algorithm, the particle achieves the search by taking determined certain line within a limited search space. And the velocity and position of each particle will restricted by various rail. It likes a flock of birds in the sky, which will reduce the flying speed and shorten the flight distance because of various external factors in search food. And the PSO algorithm can not guarantee that the probability is 1 for the global optimal solution. But in the quantum world, the moving paths of the particles are not be restricted, and are evolved by the time. The evolution process complies with the equation of Schrodinger, so the velocity and position of each particle can not be determined simultaneously. In the PSO system, if the single particle has the quantum behavior, the PSO algorithm will perform the operation according to the given rules. In the PSO algorithm, the quantum state of a particle is described by the wave function $\psi(x, t)$. When the wave function $\psi(x, t)$ is determined, the average value of any mechanical quantity and distribution of measured value of each particle is completely determined. So Sun proposed a quantum particle swarm optimization (QPSO) algorithm, the performance of the QPSO algorithm is far superior to the general PSO algorithm.

The QPSO algorithm defines the particle in the determined one quantum space of the probability density function. The state of each particle is no longer expressed by the velocity and position. The quantum state is converted into normal state the conversion of Carlo Monte method. The updating equation is described:

$$
\left\{\begin{array}{l}
x_{i}(t+1)=p_{t} \pm \alpha^{*} \mid \text { Mbest }_{i}-x_{i}(t) \mid * \ln \frac{1}{\mu} \\
p_{t}=\frac{a^{*} \text { pbest }_{t}+b^{*} \text { gbest }_{t}}{a+b} \\
\alpha=\frac{\left(\alpha_{1}-\alpha_{2}\right)^{*}\left(T_{\max }-t\right)}{T_{\max }+\alpha_{2}} \\
\text { Mbest }_{t}=\frac{\sum_{i=1}^{M} \text { pbest }_{t}(i)}{M}
\end{array}\right.
$$

Where $\alpha$ is variable contraction factor of $t$, the value of $\alpha$ is changed with the value of $t$. The $\alpha$ is used to control the speed of convergence. $\alpha_{1}$ and $\alpha_{2}$ are the starting value and the end value of variable contraction factor $\alpha . T_{\max }$ is the maximum number of iteration. In general, $\alpha_{1}=2.5, \alpha_{2}=0.5$, so the value of $\alpha$ is [0.5,2.0] for most cases. Mbest $t_{t}$ is the average of the local search optimal position of each particle in the population. $p_{t}$ is random position vector between pbest $_{t}$ and gbest $_{t}, M$ is the number of particles in the population. pbest $t_{t}$ is position vector of the searched optimal solution of the $r^{\text {th }}$ particle. $t$ is the number of the 
current iteration, $a, b, \mu$ are random number [0,1]. In the iterative process, " \pm "is determined by the random number [0,1], when the random number is greater than 0.5 , + is selected, the rest takes "-".

\section{A SAQPSO Algorithm Based on SA and QPSO Algorithm}

Although the global search ability of QPSO algorithm is stronger than the standard PSO algorithm, the QPSO algorithm is same as the standard PSO algorithm. With the advancement of evolution, the QPSO algorithm is influenced by the random vibration in the later stage, it need longer search time nearby the global optimal value, and it takes on slow convergence speed, easily traps in local minima and reduces the accuracy. The SA algorithm can greatly improve the performance of the system, increase the information processing and improve the operation speed. So the thought of simulated annealing is introduced into QPSO algorithm in order to propose an improved QPSO (SAQPSO) algorithm based on combining their respective advantages and abandoning their own shortcomings in this paper. In the proposed SAQPSO algorithm, the simulated annealing mechanism is added into the updating process of the velocity and position of each particle. The fitness of evolved population accept the optimal solution according to Metropolis criterion and the worse solution according to the probability in order to jump out the region of local extremum, adaptively adjust the annealing temperature. When the temperature gradually decreased, the particles gradually formed the low energy state and convergence to the global optimal solution. So the proposed SAQPSO algorithm makes up for falling into local optimum of PSO algorithm, and the Metropolis criterion of SA is introduced into PSO algorithm to increase the diversity of particle swarm. The particles can enter the other areas of the solution space to continue to search for the global optimal solution.

The steps of the proposed SAQPSO algorithm is described as follows:

Step1. Initialize parameters

Population size ( $M$ ), the cognition learning factor $\left(c_{1}\right)$,the social learning factor $\left(c_{2}\right)$, the number of maximum iteration $\left(T_{\max }\right)$, the starting value and the end value of variable contraction factor $\alpha_{1}$ and $\left.\alpha_{2}\right)$, random number $(a, b, \mu)$, the initial temperature $\left(T A_{0}\right)$,temperature cooling $\operatorname{system}\left(T A_{\max }\right)$, annealing $\operatorname{rate}(\lambda)$.

Step 2. Calculate the fitness value

According to solving objective optimization problem, the fitness value of each individual is calculated by the defined evaluation function. The best position of particle is pbest and global best of population is gbest.

Step 3. Calculate the value of Mbest .

The average value (Mbest) of the best position of each individual is calculated and the position of each individual is updated according to expression (5) .

Step 4. Calculate the value of particle of the next generation.

Step 5. Calculate the objective function value of each particle. And the values of gbest and pbest are updated promptly.

Step 6. Calculate the caused variation $(\Delta E)$ of fitness value by the two positions. If the $\operatorname{variation}(\Delta E)$ is less than zero or $\exp \left(\frac{-\Delta E}{T}\right)$ is greater than $\eta$ (a random number $[0,1]$ ), then a new position is accepted. Otherwise, the old position is kept.

Step 7. Reduce the temperature ( $T$ ), the cooling formula is $T=\lambda * T$.

Step 8. Determine whether the termination condition is meet. If the termination condition is meet, go to Step 9. Otherwise, return Step 2. 
Step 9. output the obtained optimal solution, and the proposed SAQPSO algorithm is end.

\section{Experiment Analysis}

In order to test the performance of the proposed SAQPSO algorithm in solving complex optimization problems, some famous benchmark functions are selected in this paper. And the PSO algorithm and QPSO algorithm are select to compare with the proposed SAQPSO algorithm. The parameters of the PSO algorithm, QPSO algorithm and SAQPSO algorithm are selected after testing. The selected ones are those that gave the best computational results concerning both the quality of the solution and the run time needed to achieve this solution. The parameters of three algorithms are given in Table 1. The experiment environment is: the Pentium IV, 4.0GB RAM, Matlab 7.8. These algorithms are independently run 20. Famous benchmark function expressions are shown Table 2.

Table 1. The Parameters of These Algorithms

\begin{tabular}{lccc}
\hline \multicolumn{1}{c}{ Parameter } & PSO & QPSO & SAQPSO \\
\hline Population size $(M)$ & 50 & 50 & 50 \\
Iteration times $\left(T_{\max }\right)$ & 1000 & 5000 & 1000 \\
Inertia weight $(w)$ & 0.85 & 0.85 & 0.85 \\
Max velocity $(V)$ & 150 & 150 & 150 \\
Cognition learning factor $\left(c_{1}\right)$ & 1.8 & 1.8 & 1.8 \\
Social learning factor $\left(c_{2}\right)$ & 1.8 & 1.8 & 1.8 \\
Starting value of variable contraction factor $\left(\alpha_{1}\right)$ & N/A & 2.0 & 2.0 \\
End value of variable contraction factor $\left(\alpha_{2}\right)$ & N/A & 0.5 & 0.5 \\
Initial temperature $\left(T A_{0}\right)$ & N/A & N/A & 30 \\
Temperature cooling $\left(T A_{\max }\right)$ & N/A & 7N/A & 1000 \\
Annealing rate $(\lambda)$ & N/A & 7N/A & 0.8 \\
\hline
\end{tabular}

Table 2. Benchmark Function Expressions (30D)

\begin{tabular}{cccc}
\hline Function & Function Expression & $\begin{array}{c}\text { Optimal } \\
\text { value }\end{array}$ & $\begin{array}{c}\text { Variable } \\
\text { range }\end{array}$ \\
\hline$F_{1}$ & $F(x)=\max _{i}\left\{\left|x_{i}\right|, 1 \leq i \leq n\right\}$ & $\mathbf{0}$ & {$[-\mathbf{- 1 0 0 , 1 0 0}]$} \\
$F_{2}$ & $F(x)=\sum_{i=1}^{n-1} 100\left(x_{i+1}-x_{i}^{2}\right)^{2}+\left(x_{i}-1\right)^{2}$ & $\mathbf{0}$ & {$[-\mathbf{3 0 , 3 0}]$} \\
$F_{3}$ & $F(x)=-20 \exp \left(-0.2 \sqrt{\left.\sum_{i=1}^{n} \frac{x_{i}^{2}}{n}\right)-\exp \left(\sum_{i=1}^{n} \cos \left(2 \pi x_{i}\right) / n\right)+20+e}\right.$ & $\mathbf{0}$ & {$[-\mathbf{- 3 2 , 3 2}]$} \\
$F_{4}$ & $F(x)=\frac{1}{4000} \sum_{i=n}^{n} x_{i}^{2}-\prod_{i=1}^{n} \cos \left(\frac{x_{i}}{\sqrt{i}}\right)+1$ & $\mathbf{0}$ & {$[-\mathbf{- 6 0 0 , 6 0 0}]$}
\end{tabular}




$$
F_{5} \quad F(x)=\sum_{i=1}^{n}\left(x_{i} \sin \sqrt{\left|x_{i}\right|}\right)
$$

Table 3. The Experimental Results (30D)

\begin{tabular}{ccccc}
\hline Function & Opt. value & Algorithm & Min value & Average value \\
\hline \multirow{2}{*}{$F_{1}$} & 0 & PSO & $4.013451 \mathrm{E}-003$ & $6.264106 \mathrm{E}-003$ \\
& & QPSO & $2.813326 \mathrm{E}-014$ & $8.012349 \mathrm{E}-013$ \\
$F_{2}$ & SAQPSO & $2.813326 \mathrm{E}-033$ & $5.850461 \mathrm{E}-033$ \\
\hline \multirow{2}{*}{$F_{3}$} & PSO & $1.471427 \mathrm{E}-000$ & $1.172530 \mathrm{E}+001$ \\
& & QPSO & $5.147341 \mathrm{E}-003$ & $2.531367 \mathrm{E}-002$ \\
& & SAQPSO & $6.257481 \mathrm{E}-007$ & $4.750601 \mathrm{E}-005$ \\
\hline \multirow{2}{*}{$F_{3}$} & PSO & $3.103485 \mathrm{E}-007$ & $3.407652 \mathrm{E}-006$ \\
$F_{4}$ & QPSO & $4.134249 \mathrm{E}-011$ & $5.461460 \mathrm{E}-011$ \\
& & SAQPSO & $1.930702 \mathrm{E}-016$ & $3.401956 \mathrm{E}-015$ \\
\hline \multirow{2}{*}{$F_{5}$} & PSO & $5.530146 \mathrm{E}-004$ & $4.093296 \mathrm{E}-003$ \\
& & QPSO & $2.508642 \mathrm{E}-018$ & $9.075326 \mathrm{E}-018$ \\
& & SAQPSO & $1.180482 \mathrm{Ee}-036$ & $4.530492 \mathrm{E}-033$ \\
\hline
\end{tabular}

As can be seen from the Table 3, for selected 5 benchmark functions, the proposed SAQPSO algorithm can obtain the best solution by observing and analyzing the experiment results. That's to say, the proposed SAQPSO algorithm takes on better optimization performance than the PSO algorithm and QPSO algorithm in solving $F_{1}$, $F_{2}, F_{3}, F_{4}$ and $F_{5}$. And the QPSO algorithm takes on better optimization performance than the PSO algorithm in solving $F_{1}, F_{2}, F_{3}, F_{4}$ and $F_{5}$. For $F_{4}$ function, the proposed SAQPSO algorithm is near to the optimal value (zero). So the experiment results show that the proposed SAQPSO algorithm is more capable to solve the global optimization problems and overcome the premature phenomenon.

\section{Conclusion}

The PSO algorithm is one of swarm intelligence algorithms, it is also a branch of evolutionary computation. After the random solutions are initialized by the system, the PSO algorithm can search for the optimal value by the iteration. It constantly searches for the global optimal solution by updating the velocity and position of particles. In the application of the PSO algorithm, it has some disadvantages of the low computational precision, local optimal solution and slow convergence speed. So the SA algorithm and quantum theory are introduced into the PSO algorithm in order to improved PSO(SAQPSO) algorithm for improving the search speed and the convergence precision and guaranteeing the simplification and effectiveness, keeping the diversity of the population, avoiding falling into local optimum and enhancing the global search ability. Finally, five benchmark functions with 30 dimensions are selected to test the performance of the SAQPSO algorithm. The 
results show the proposed MSCQPSO algorithm can execute the optimization calculations, and obtain the best solution than the PSO algorithm and QPSO algorithm. The proposed SAQPSO algorithm takes on the fast convergence speed, the better robustness and global search ability.

\section{Acknowledgements}

This research was supported by the National Natural Science Foundation, China (No.51175187) and the Science \& Technology Research Program of Guangdong Province, China (No.2013B010401041) , and the Science \& Technology Research Program of Dongguan City, China (2014509102211).

\section{References}

[1] J. Kennedy and R. C. Eberhart, "Particle swarm optimization", Proc IEEE Intentional Conference on Neural Networks. Piscataway, NJ: IEEE Press, (1995), pp. 1942-1948.

[2] F. Van den Berg, "Particle swarm weight initialization in multi-layer perception artificial neural networks", Proc of Development and Practice Artificial Intelligence Techniques, Durban, South Africa, (1999), pp. 41-45.

[3] G. Ciuprina, D. Ioan and I. Munteanu, "Use of intelligent particle swarm optimization in electromagnetics", IEEE Trans. on Magnetics, vol. 38, no. 2, (2002), pp. 1037-1040.

[4] L. Xu, J. H. Wang, Y. L. Yan and S. S. Gu, "Improved PSO and its application to load distribution optimization of hot strip mills", Kongzhi yu Juece/Control and Decision, vol. 20, no. 12, (2005), pp. 1379-1383.

[5] X. J. Jiang, X. G. Xiong and Y. W. Wu, "Improved PSO algorithm and its application in short-term generation scheduling", Dianli Zidonghua Shebei / Electric Power Automation Equipment, vol. 25, no. 3, (2005), pp. 37-40.

[6] Y. Q. Luo, X. G. Yuan, Y. J. Liu, "An improved PSO algorithm for solving non-convex NLP/MINLP problems with equality constraints", Computers and Chemical Engineering, vol. 31, no. 3, (2007), pp. 153-162.

[7] L. Y. Chuang, H. W. Chang, C. J. Tu and C. H. Yang, "Improved binary PSO for feature selection using gene expression data", Computational Biology and Chemistry, vol. 32, no. 1, (2008), pp. 29-37.

[8] X. H. Yuan, A. J. Su, Y. B. Yuan, H. Nie and L. Wang, "An improved PSO for dynamic load dispatch of generators with valve-point effects”, Energy, vol. 34, no. 1, (2009), pp. 67-74.

[9] P. K. Hota, A. K. Barisal and R. Chakrabarti, "An improved PSO technique for short-term optimal hydrothermal scheduling”, Electric Power Systems Research, vol. 79, no. 7, (2009), pp. 1047-1053.

[10] S. N. Omkar, R. Khandelwal, T. V. S. Ananth, G. N. Naik and S. Gopalakrishnan, "Quantum behaved Particle Swarm Optimization (QPSO) for multi-objective design optimization of composite structures", Expert Systems with Applications, vol. 36, no. 8, (2009), pp. 11312-11322.

[11] T. Niknam, B. Amiri, J. Olamaei and A. Arefi, "An efficient hybrid evolutionary optimization algorithm based on PSO and SA for clustering”, Journal of Zhejiang University: Science A, vol. 10, no. 4, (2009), pp. 512-519.

[12] J. Behnamian and S. M. T. Fatemi Ghomi, "Development of a PSO-SA hybrid metaheuristic for a new comprehensive regression model to time-series forecasting", Expert Systems with Applications, vol. 37, no. 2, (2010), pp. 974-984.

[13] S. F. Dong, Z. C. Dong, J. J. Ma and K. N. Chen, "Improved PSO algorithm based on chaos theory and its application to design flood hydrograph", Water Science and Engineering, vol. 3, no. 2, (2010), pp. 156-165.

[14] R. F. Abdel-Kader, "An improved discrete PSO with GA operators for efficient QoS-multicast routing", International Journal of Hybrid Information Technology, vol. 4, no. 2, (2011), pp. 23-38.

[15] Y. Wang, Z. Jing, Z. Chao, Y. Q. Wang, Q. Hui and Y. L. Lu, "An improved self-adaptive PSO technique for short-term hydrothermal scheduling”, Expert Systems with Applications, vol. 39, no. 3, (2012), pp. 2288-2295.

[16] A. Tajbakhsh, K. Eshghi and A. Shamsi, "A hybrid PSO-SA algorithm for the travelling tournament problem", European Journal of Industrial Engineering, vol. 6, no. 1, (2012), pp. 2-25.

[17] K. Thanushkodi and K. Deeba, "On performance analysis of hybrid intelligent algorithms (Improved PSO with SA and Improved PSO with AIS) with GA, PSO for multiprocessor job scheduling”, WSEAS Transactions on Computers, vol. 11, no. 5, (2012), pp. 131-147.

[18] X. Gao, Q. H. Wan, S. W. Yang, W. Chen and C. H. Zhao, "Improved PSO algorithms for improving the subdivision accuracy of photoelectric rotary encoder", Hongwai yu Jiguang Gongcheng/Infrared and Laser Engineering, vol. 42, no. 6, (2013), pp. 1508-1513. 
[19] O. A. Behrooz, M. Pooya and S. P. Masoud, "An improved PSO algorithm with a territorial diversitypreserving scheme and enhanced exploration-exploitation balance", Swarm and Evolutionary Computation, vol. 11, (2013), pp. 1-15.

[20] Y. L. Li, W. Shao, L. You and B. Z. Wang, "An improved PSO algorithm and its application to UWB antenna design", IEEE Antennas and Wireless Propagation Letters, vol. 12, (2013), pp. 1236-1239.

[21] E. Walid, E. A. Haikal, A. Abraham and A. M. Alimi, "A comparative study of the improvement of performance using a PSO modified by ACO applied to TSP", Applied Soft Computing Journal, vol. 25, no. 12, (2014), pp. 234-241.

[22] W. D. Ji, J. H. Wang and J. Zhang, "Improved PSO based on update strategy of double extreme value", International Journal of Control and Automation, vol. 7, no. 2, (2014), pp. 231-240.

[23] G. G. Chen, L. L. Liu, P. Z. Song and Y. W. Du, "Chaotic improved PSO-based multi-objective optimization for minimization of power losses and L index in power systems", Energy Conversion and Management, vol. 86, (2014), pp. 548-560.

[24] T. Wu, Y. S. Yan and X. Chen, "Improved dual-group interaction QPSO algorithm based on random evaluation", Kongzhi yu Juece/Control and Decision, vol. 30, no. 3, (2015), pp. 526-530.

[25] S. Kirkpatric, J. C. D. Gelatt and M. P. Vecchi, "Optimization by simulated annealing", Science, no. 220, (1983), pp. 671-680.

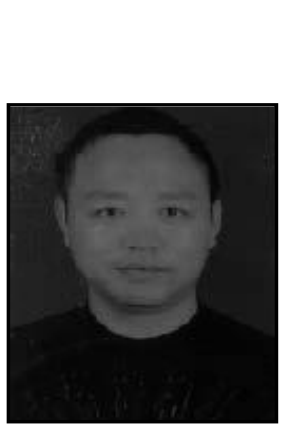

\begin{abstract}
Authors
Wei Tan, he was born in 1974, $\mathrm{PhD}$. associate professor. He graduated from the College of mechanical and automotive engineering, South China University of Technology and got $\mathrm{PhD}$ in 2013. He is now a postdoctoral researcher at School of computer science and engineering in South China University of Technology. He has published more than 10 papers indexed by SCI/EI in domestic and foreign academic journals and international academic conferences. At present, his research interests are in service computing, cloud computing, cloud manufacturing, swarm intelligence.
\end{abstract}

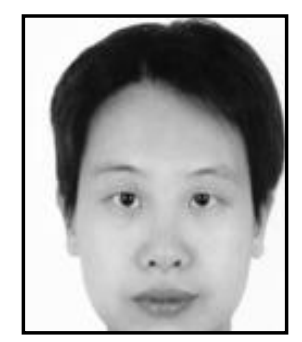

Shoubin Dong, he was born in 1967, PhD, Professor, doctoral supervisor. She is deputy director of the information network engineering research center of South China University of Technology, vice director of computer network Key Laboratory of Guangdong Province, and is a leader for South China University of Technology high performance computing and massive information processing academic team. In 1994, she graduated and got Ph.D. from the University of Science \&Technology China, Department of information science and Electronic Engineering. She has published more than 60 papers indexed by SCI/EI in domestic and foreign academic journals and international academic conferences

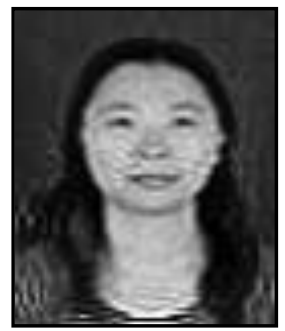

Xuan Liu, she was born in 1975, Master. She is graduated from the HUNAN University in 2013.She has published more than 6 papers indexed by SCI/EI in domestic and foreign academic journals and international academic conferences. At present, her research interests are in service computing, Accounting Informationization. 


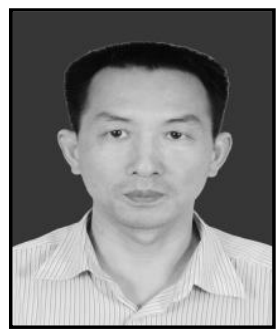

Bin Wang, he was born in 1964, Master. He is graduated from the Wuhan University in 1989 .He has published more than 10 papers indexed by SCI/EI in domestic and foreign academic journals and international academic conferences. At present, his research interests are in software engineering. 